\title{
Efeitos da Expansão do Biodiesel e da Troca de Fontes Triglicerídeas no Cenário Energético Brasileiro
}

\author{
Gerd Brantes Angelkorte \\ Programa de Planejamento Energético (PPE), Instituto Alberto Luiz Coimbra de Pós-Graduação e Pesquisa em Engenharia \\ (COPPE) - Universidade Federal do Rio de Janeiro (UFRJ), Rio de Janeiro, Brasil \\ Email: angelkorte@ppe.ufrj.br
}

\begin{abstract}
Resumo
A preocupação com os efeitos dos impactos ambientais proporcionados pelo aquecimento global tem feito a população mundial buscar novas fontes energéticas menos agressivas ao meio ambiente. Com isso, o biodiesel vem ganhando força e tem expandido sua proporção na mistura com o diesel. Contudo, o Brasil ainda utiliza cerca de $20 \%$ de sebo bovino, que emite grande quantidade de GEE, degrada o solo e apresenta grande consumo de água. O presente trabalho buscou avaliar as possibilidades e os efeitos da substituição dessa fonte não renovável por outras de origem vegetal, assim como os efeitos ambientais do aumento da porcentagem de biodiesel, chegando a patamares de $20 \%$ e $30 \%$. Para isso, produziram-se e testaram-se dois tipos de biodieseis, com e sem sebo bovino, posteriormente foram utilizados os resultados obtidos e dados da frota a diesel para modelar, com o auxílio do modelo MoMo Lite, os impactos e emissões de $\mathrm{CO}_{2 \mathrm{eq}}$ no Brasil. Conseguiu-se determinar o grande benefício da adoção de maiores teores de biodiesel no diesel, principalmente quando há a substituição do sebo bovino por fontes vegetais, além da importância da adoção de análises mais amplas de todo o ciclo de produção da matéria-prima. Esses resultados variaram apenas $10 \%$, ao observar apenas os dados de emissão de $\mathrm{CO}_{2 \mathrm{eq}}$ na queima, porém ao analisar os resultados através do well-totank, essa variação subiu para cerca de $52 \%$.
\end{abstract}

Palavras-chave: biodiesel, fontes renováveis, bioenergia, modelagem, MoMo.

\section{Effects of Biodiesel Expansion and the Exchange of Triglyceride Sources in the Brazilian Energy Scenario}

\begin{abstract}
The concern with global warming impacts on the environment has made the world population search for new energy sources that are less aggressive to the environment. Therefore, biodiesel has become more relevant and has expanded its proportion in the blend with diesel. However, Brazil still uses about $20 \%$ of bovine tallow, which emits large amount of GHG, degrades the soil and entails great water consumption. The purpose of this study was to evaluate the possibilities and effects of the substitution of this nonrenewable source for others of vegetable origin, as well as the environmental effects of increasing the percentage of biodiesel, reaching levels of $20 \%$ and $30 \%$. Hence, two types of biodiesel were produced and tested, with and without bovine tallow, and the results obtained and data from the diesel fleet were used to model the impacts and $\mathrm{CO}_{2 \mathrm{eq}}$ emissions with the aid of the MoMo Lite model in Brazil. It was possible to determine the great benefit of adopting higher levels of biodiesel in diesel (especially when there was a substitution of bovine tallow for plant sources), besides the importance of adopting broader analysis of the whole production cycle of the raw material. Since only $\mathrm{CO}_{2 \mathrm{eq}}$ emission data were observed at the burning, the results varied only $10 \%$, but when the results were analyzed through the well-to-tank, this variation rose to $52 \%$.
\end{abstract}

Keywords: biodiesel, renewable sources, bioenergy, modeling, MoMo.

\section{Introdução}

Devido à crescente preocupação mundial com o meio ambiente, a percepção de que a humanidade também está inserida no contesto ambiental e que toda a degradação da natureza acaba impactando também na vida do ser humano, o homem vem procurando soluções para diminuir e mitigar os danos provocados pelos gases de efeito estufa (GEE) que estão causando o aquecimento do planeta.

Cita: Brantes Angelkorte, G. (2019). Efeitos da Expansão do Biodiesel e da Troca de Fontes Triglicerídeas no Cenário Energético Brasileiro. Revista Fuentes: El reventón energético, 17(2), 87-99. 
Nos últimos anos o biodiesel tem ganhado cada vez mais espaço no cenário energético com os constantes aumentos de sua proporção obrigatória no diesel, passando de 7\% em 2016 para 10\% em março de 2018 (ANP, 2019). Em 2003, quando o Programa Nacional de Produção e Uso de Biodiesel - PNPB começou, o teor de $2 \%$ era voluntário, funcionando como meio de estimular a permanência no campo do pequeno produtor rural, evitando a migração da população para os centros urbanos e levando dignidade e desenvolvendo para a região. Logo possuindo um viés mais social do que realmente como forma de mitigar os problemas ambientais causados pelas emissões de GEE veiculares.

Esse percentual facultativo permaneceu assim até janeiro de 2008, quando se tornou obrigatório, porém, com a expansão do volume de biodiesel para o abastecimento da frota brasileira, o contexto social do PNPB passou a não ser mais uma verdade, visto que a matéria-prima básica para a produção de biodiesel passou a ser a soja e o sebo bovino (BRASIL, 2016). Porém, com os latifúndios, grandes problemas ambientais causados principalmente devido à expansão da pecuária e das fronteiras agrícolas começaram a surgir.

Apesar de haver impactos ambientais referentes as fontes de matéria-prima de origem vegetal, elas possuem vantagens sobre as de origem animal, como a retirada de dióxido de carbono $\left(\mathrm{CO}_{2}\right)$ da atmosfera durante sua fase de desenvolvimento. No caso das leguminosas, como a soja, ainda há a fixação de nitrogênio $\left(\mathrm{N}_{2}\right)$ através da planta durante o seu crescimento celular. Por sua vez, os bovinos são responsáveis pela liberação de uma grande quantidade de GEE durante o processo de ruminação e digestão de alimentos. Ainda, prejudicam a estrutura solo durante o pisoteamento, fazendo com que ele seja compactado, auxiliando no processo de erosão do solo, visto que ele já encontrasse exposto devido a retirada da vegetação nativa para a introdução de gramíneas.

Além do fato da criação de bovinos trazer grandes impactos por si só devido a degradação do solo e emissões de GEE, a humanidade vem discutindo nos últimos anos a real necessidade de uma alimentação tão fortemente baseada na ingestão de proteínas de origem animal, devido a fatores ambientais e de saúde. Logo, se torna inevitável que nos próximos anos comece a haver uma mudança significativa na dieta alimentar mundial (IPCC, 2019), o que poderá afetar o fornecimento de uma das principais fontes de matéria-prima do setor de biodiesel no Brasil que é o sebo bovino.

\section{Objetivo}

O estudo teve como objetivo a modelagem através do modelo Mobility Model Lite - MoMo Lite, de uma política de expansão da porcentagem de biodiesel presente no diesel de petróleo, assim como da troca do sebo bovino como fonte de matéria-prima por outras fontes triglicerídeas de origem vegetal como o óleo de semente de algodão. Com isso, avaliar os impactos ambientais através do cálculo da emissão direta da queima do biocombustível e da análise wellto-tank.

\section{Revisão bibliográfica}

\section{Biodiesel}

O primeiro motor ciclo diesel foi desenvolvido por Rudolf Diesel (1838-1913) para ser exposto e apresentado para a sociedade na Feira Mundial de Paris de 1900, vindo a funcionar plenamente com óleo de amendoim (NITSKE e WILSON, 1965).

Porém, foi apenas após o período de instabilidade do mercado do petróleo, com os choques da década de 1970, que a pesquisa e a utilização de biocombustíveis como o biodiesel tomou força, mostrando-se uma possibilidade para redução da dependência do petróleo exterior e permitindo assim, maior segurança energética (COSTA, 2017).

Segundo a Agência Nacional de Petróleo, Gás Natural e Biocombustíveis - ANP (2016), o biodiesel é um combustível composto de alquil ésteres de ácidos, carboxílicos de cadeia longa, produzido a partir do processo de transesterificação ou esterificação de materiais graxos de origem animal ou vegetal, e que atendam as especificações contidas no Regulamento Técnica $n^{\circ} 3 / 2014$, da Resolução ANP no 45 de 25 de agosto de 2014 .

O biodiesel é formado através da reação química, denominada de transesterificação, no qual, os triglicerídeos/ésteres de ácidos graxos, que são os maiores componentes dos óleos vegetais e das gorduras animais, reagem com o catalizador e o álcool para produzir biodiesel e glicerina, que é um composto indesejado, visto ao seu menor valor comercial, Figura 1. As rotas mais utilizadas para esse processo são as rotas etílicas e metílicas, sendo a segunda mais utilizada devido ao seu menor custo (KNOTHE et al., 2006). 


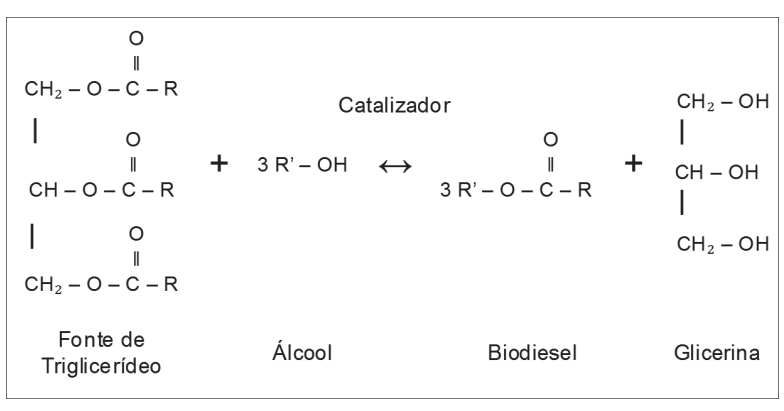

Figura 1. Processo de transesterificação (Adaptado de KNOTHE et al., 2006)

Como a produção de biodiesel necessita de uma fonte triglicerídea qualquer para o processo de transesterificação, sendo possível determinar uma vasta gama de possíveis fontes de matérias-primas, incluindo óleos vegetais, como o de soja, caroço de algodão, coco e palma, gorduras animais, como o caso do sebo bovino e de frango, e também, óleos de descarte, como é o exemplo do óleo usado para frituras.

Segundo Knothe et al. (2006), o biodiesel possui perfeita miscibilidade com o petrodiesel em qualquer proporção e possui vantagens como, por exemplo, derivar de matérias-primas renováveis, ser biodegradável, gerar uma menor quantidade de gases causadores do efeito estufa ao ser queimado, como o caso dos $\mathrm{CO}_{\mathrm{X}}$, possuir um alto ponto de fulgor, aumentando a segurança no caso de armazenamento e manuseio do mesmo e apresentar uma boa lubricidade, o que no caso de misturas com o petrodiesel, acaba auxiliando na lubricidade do mesmo e agindo portanto, como um aditivo (AYALA, 2011). Porém, o biodiesel apresenta algumas desvantagens como o elevado custo de produção, o aumento da emissão de gases do tipo $\mathrm{NO}_{\mathrm{X}}$ devido a queima no processo de combustão, o elevado grau de oxidação, quando exposto ao ar e o conflito alimentação versus combustível, que ocorre devido a utilização de matériasprimas que anteriormente seriam destinadas para o setor de alimentação e passam a ser empregadas no setor energético (VILLELA, 2014).

Atualmente, o biodiesel possui uma grande importância no cenário mundial. Em países da Europa, como a Alemanha, ele já é utilizado em concentração de $20 \%$ em mistura com o petrodiesel (B20), já nos EUA, as proporções variam de $2 \%$ até $100 \%$ de biodiesel na mistura. No Brasil, a porcentagem adotada nas bombas de combustível, atualmente é de $10 \%$.

Outro passo importante para a expansão das fronteiras do biodiesel no Brasil foi a Resolução no 3 de 21/09/2015 do Conselho Nacional de Política Energética - CNPE, que autorizou e definiu diretrizes para a comercialização e o uso voluntário de biodiesel. Em que, no seu art. $1^{\circ}$, a CNPE autorizou a comercialização e o uso voluntário de misturas superiores, chegando ao patamar de B20 para frotas cativas ou para consumidores rodoviários, B30 para transporte ferroviário e uso agrícola e industrial e B100 para o uso experimental, específico ou nas demais aplicações. Sendo esse uso regulamentado pela ANP $n^{\circ}$ 30 de 23 de junho de 2016.

\section{Fontes de triglicerídeo}

No mundo, as matérias-primas mais utilizadas para a produção de biodieseis são os óleos vegetais e isso, graças à grande disponibilidade e variedade deles. $\mathrm{O}$ PNPB foi desenvolvido pensando-se justamente nessa vasta quantidade de fontes triglicerídeas estimulando a utilização de diferentes oleaginosas, de forma a aproveitar os potenciais produtivos de cada região e tentando (COSTA, 2017). Porém, as fontes de origem animal, como o sebo bovino, também possuem uma grande importância para a produção de biodiesel, visto a principalmente seu baixo custo, já que ela é um resíduo que anteriormente seria descartada (URIBE, 2014).

Essa grande diversidade de matérias-primas pode ser percebida quando se compara as fontes oleaginosas mais utilizadas em cada região/país. Na União Europeia (UE), os óleos de colza e girassol se destacam como os mais utilizados (HAROLD, 1997), já nos países tropicais, o óleo de palma é o predominante em industrias de biodiesel (MASJUKI; SAPUAN, 1995), e por sua vez, o óleo de soja e as gorduras animais são os que representam as principais matérias-primas nesse setor nos EUA e no Brasil (JEWETT, 2003).

Pode-se também inferir outros fatores para a escolha da melhor fonte oleaginosa para a produção de biodiesel, sendo eles, o custo e a disponibilidade, como já explicitados, as propriedades de armazenamento, o desempenho como combustível e as decisões governamentais, em forma de subsídios estabelecidos por programas nacionais (AMARIS, 2015). Com isso, esses fatores podem acabar tendo um grau de influência muito elevado para a adoção de uma determinada fonte de matéria-prima (SINGH; SINGH, 2010).

Outro ponto levantado por Singh e Singh (2010) é o fato de cada fonte produzir um biodiesel diferente, o que pode acabar sendo benéfico ou prejudicial para o produto. Pode-se perceber isso principalmente quando comparamos duas características muito importante para esse tipo de combustível, que são a viscosidade cinemática à $40^{\circ} \mathrm{C}$ e o ponto de entupimento de filtro a 
frio (PEFF), que nas gorduras animais, principalmente o sebo bovino, apresentam valores muito superiores do que se comparado com outras fontes de origem vegetal, como a soja e o algodão. Sendo essas características fundamentais para a decisão de utilizar esse tipo de fonte ou não, visto que em países com temperaturas mais frias, um motor que esteja utilizando um biocombustível com PEFF superior poderá trazer problemas como perda de escoabilidade desse fluido, impossibilitando, portanto, o funcionamento desse veículo.

No Brasil, ainda há uma maciça adoção da soja como a principal fonte triglicerídea para a produção de biodiesel, sendo o sebo bovino o segundo mais utilizado e o óleo de caroço de algodão o terceiro, conforme pode se observar no Gráfico 1.
Porém, segundo previsões da Associação Brasileira de Indústrias de Óleos Vegetais - ABIOVE, até 2030 (em um cenário de B20), a participação do sebo bovino deverá cair para o patamar de $8 \%$ e no seu lugar, entrará o óleo de palma e de algodão, que assumirá $15 \%$ do mercado de biodiesel (ABIOVE; APROBIO; UBRABIO, 2016).

\section{Emissões de gases de efeito estufa}

A emissão de gases de efeito estufa (GEE) é inevitável durante o processo de combustão de qualquer combustível, porém, segundo Salomão (2013), a substituição do diesel de origem fóssil por biodiesel propicia uma significante redução de poluentes na atmosfera, principalmente de óxido de enxofre ( $\mathrm{SOx})$ e materiais particulados.

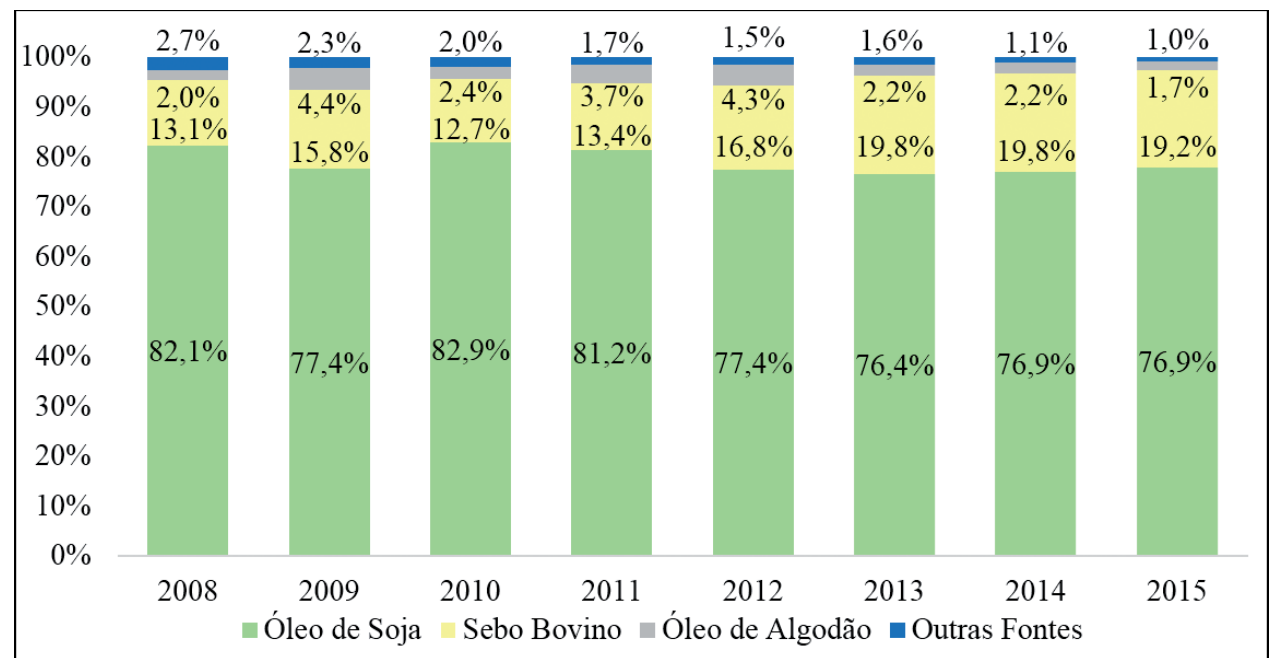

Gráfico 1. Participação das matérias-primas na produção de biodiesel no Brasil (Adaptado de BRASIL, 2016)

A redução dessas emissões além de gerar um menor impacto ambiental, no contexto de poluição atmosférica local, também, é fundamental para a saúde humana, visto que os GEE e particulados, liberados na atmosfera principalmente pela queima de combustíveis fósseis através da frota veicular, são responsáveis por inúmeras doenças de via respiratória (COSTA, 2017 e WHO, 2014). Apesar de Reijinders e Huijbregts (2008) afirmarem que a diminuição das emissões de SOx serem compensadas pelo aumento da descarga óxido nitroso (NOx) propiciada pelos biodieseis, Giakoumus (2012) contrapõe falando que a diminuição dos níveis de emissões de poluentes como hidrocarbonetos (HC), monóxido de carbono (CO) e particulados são um fator essencial para a diminuição do surgimento e agravamento de casos de doenças de vias respiratórias. Fazendo com que ainda que importante, o incremento das emissões de NOx não seja superior a diminuição dos outros fatores que a adição de biodiesel no diesel é capaz de trazer para a diminuição de doenças respiratórias.

Segundo Costa (2017), o B100 permite uma redução de até $67 \%$ de hidrocarbonetos totais, $47 \%$ de particulados e $47 \%$ de monóxido de carbono e somente um aumento de $10 \%$ dos níveis de NOx se comparado com o diesel. Entretanto, conforme exemplificado por Rosa et al. (2003), essa redução terá grande influência da fonte de triglicerídeo e da rota que lhe deu origem.

Porém, a emissão dos GEE não se dá somente durante o processo de combustão no motor, mas sim, durante toda a cadeia de produção desse combustível, que no caso dos biocombustíveis inicia-se no setor agrícola. Portanto, é muito comum utilizar a análise de ciclo 
de vida (ACV) para investigar e avaliar os impactos ambientais causados ao longo da cadeia produtiva desse combustível (CHERUBINI, 2010). Sendo que para combustíveis, é comum utilizar-se a análise wellto-wheel que tem como objetivo comparar diferentes tipos de combustíveis e suas cadeias produtivas, ou então, well-to-tank, que não inclui a etapa de consumo de combustível e é utilizado para combustíveis de mesma origem, logo, que possuam desempenhos de combustão semelhantes, que é o caso dos biodieseis (GNANSOUNOU et al., 2009).

Em análises feitas por Coronado et al. (2009), considerando ACV que quanto maior o percentual de biodiesel no diesel, menores são as emissões de $\mathrm{CO}_{2 \mathrm{eq}}$. Além disso, que biodieseis de fonte vegetal ao final da cadeia, apresentavam níveis menores de emissão do que biodieseis de fonte animal ou o diesel de petróleo, devido ao processo de fixação de carbono que ocorre durante a fotossíntese, logo, sendo o biodiesel de fonte vegetal ambientalmente melhor do que os demais.

\section{Metodologia científica}

\section{Produção do biodiesel}

O processo de produção do biodiesel foi realizado no Laboratório de Máquinas Agrícolas - LABMAQ da Universidade Federal Fluminense - UFF. Para o estudo, produziu-se dois tipos de biodiesel, sendo um denominado "Padrão", que foi produzido com $77 \%$ de óleo de soja, $19 \%$ de sebo bovino e $4 \%$ de óleo de algodão, conforme a média apresentada anteriormente no Gráfico 1, e o outro, denominado "Sem Sebo Bovino", que foi produzido com $85 \%$ de óleo de soja e $15 \%$ de óleo de algodão.

O processo utilizado para a produção do biodiesel foi o de transesterificação por agitação magnética à $45^{\circ} \mathrm{C}$ com pré-aquecimento à $45^{\circ} \mathrm{C}$ por rota metílica na proporção molar de $1 \mathrm{~mol}$ de óleo para 6 mols de metanol P.A. 99,7\%, através de catálise alcalina com hidróxido de sódio (TOMASEVIC; MARINKOVIC, 2003).

Após o processo de produção do biodiesel, utilizouse diesel S10 (diesel com 10 ppm de enxofre em sua composição) sem aditivos doado pela empresa Ipiranga para se fazer a mistura com o biodiesel, produziu-se os combustíveis (Tabela 1).
Tabela 1. Combustíveis desenvolvidos para o estudo (Fonte: Elaboração própria).

\begin{tabular}{|l|l|}
\hline \multicolumn{1}{|c|}{ Combustível } & \multicolumn{1}{|c|}{ Descrição } \\
\hline BO & $100 \%$ diesel S10 \\
\hline B8 Padrão & $\begin{array}{l}92 \% \text { diesel S10 }+8 \% \text { biodiesel (soja + sebo } \\
\text { bovino + algodão) }\end{array}$ \\
\hline B8 Sem Sebo Bovino & $\begin{array}{l}92 \% \text { diesel S10 }+8 \% \text { biodiesel (soja }+ \\
\text { algodão) }\end{array}$ \\
\hline B10 Padrão & $\begin{array}{l}90 \% \text { diesel S10 }+10 \% \text { biodiesel (soja }+ \\
\text { sebo bovino + algodão) }\end{array}$ \\
\hline B10 Sem Sebo Bovino & $\begin{array}{l}90 \% \text { diesel S10 }+10 \% \text { biodiesel (soja }+ \\
\text { algodão) }\end{array}$ \\
\hline B20 Padrão & $\begin{array}{l}80 \% \text { diesel S10 }+20 \% \text { biodiesel (soja }+ \\
\text { sebo bovino + algodão) }\end{array}$ \\
\hline B20 Sem Sebo Bovino & $\begin{array}{l}80 \% \text { diesel S10 }+20 \% \text { biodiesel (soja }+ \\
\text { algodão) }\end{array}$ \\
\hline B30 Padrão & $\begin{array}{l}70 \% \text { diesel S10 }+30 \% \text { biodiesel (soja }+ \\
\text { sebo bovino }+ \text { algodão) }\end{array}$ \\
\hline B30 Sem Sebo Bovino & $\begin{array}{l}70 \% \text { diesel S10 }+30 \% \text { biodiesel (soja }+ \\
\text { algodão) }\end{array}$ \\
\hline B100 Padrão & $\begin{array}{l}100 \% \text { biodiesel (soja }+ \text { sebo bovino }+ \\
\text { algodão) }\end{array}$ \\
\hline B100 Sem Sebo Bovino & $100 \%$ biodiesel (soja + algodão) \\
\hline
\end{tabular}

\section{Caracterização físico-química}

A caracterização físico-química dos biocombustíveis produzidos foi feita no Laboratório de Reologia LARE da UFF, sendo feitos os estudos da viscosidade cinemática à $40^{\circ} \mathrm{C}$ pelo método do viscosímetro de capilar do tipo Cannon-Fenske, e da massa específica à $20^{\circ} \mathrm{C}$ pelo método da picnometria de cada biocombustível produzido (ANGELKORTE, 2016).

\section{Emissão de gases de efeito estufa}

Para a análise de gases, utilizou-se um microtractor agrícola com motor monocilindro de $868 \mathrm{~cm}^{3}$ modelo M 99 ID da Agrale que fornece 10,8 kW (14,5 hp) de potência a $2750 \mathrm{rpm}$ (Figura 2) e um analisador de gases modelo $350 \mathrm{XL}$ da Testo.

O estudo foi desenvolvido com o motor operando em rotação típica de trabalho de $2000 \mathrm{rpm}$ e após o motor aquecido e operando em estado estacionário, introduziuse a sonda do analisador de gases no escapamento do motor, fazendo-se leituras constantes a cada segundo durante 120 segundos. Foram feitas três repetições para cada biocombustível, sendo que durante todas as medições também foi analisado a temperatura e umidade do ar, procurando não realizar medições com esses fatores variando mais do que $10 \%$, a fim de não haver qualquer interferência externa durante o processo de aferição dos dados. 


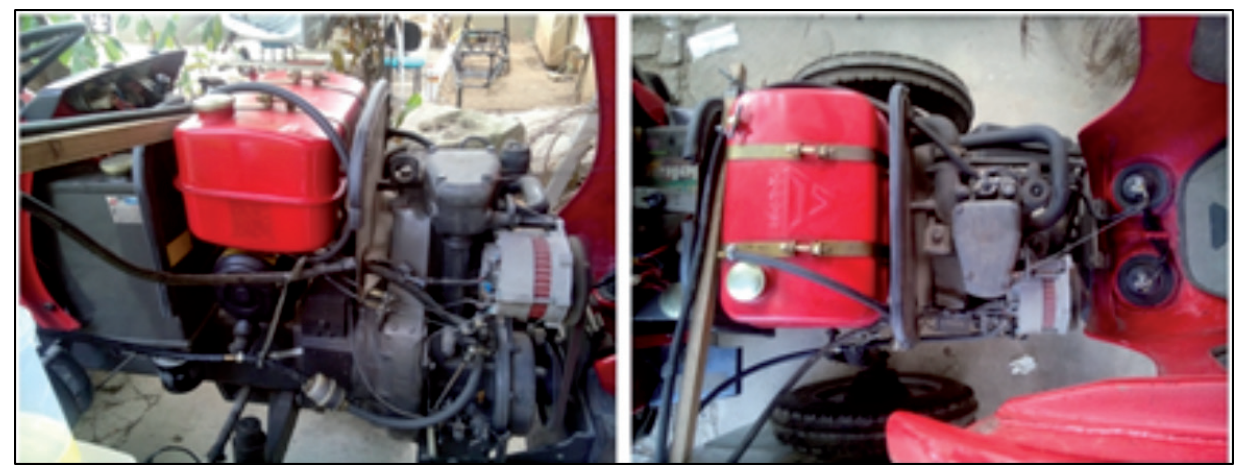

Figura 2. Microtrator agrícola Agrale (Fonte: Elaboração própria).

\section{Modelagem da evolução emissões de gases} de efeito estufa de veículos diesel no Brasil

Para a modelagem de dados, utilizou-se o modelo paramétrico Mobility Model Lite - MoMo Lite da Agência Internacional de Energia - IEA. Ele é um modelo que permite análises do tipo bottom-up e que procura desenvolver subsídios para resolver problemas do tipo what-if. O modelo foi desenvolvido de modo a representar diversos tipos de veículos automotores, fazendo projeções de consumo de combustíveis e emissões de $\mathrm{CO}_{2}$ equivalente (BARTOLO, 2012). Além disso, o presente trabalho utilizou Bartolo (2012) como referência para a parte de modelagem no MoMo Lite.

$\mathrm{Na}$ fase de modelagem foram analisadas a expansão da frota propelida por motores ciclo diesel no Brasil e da evolução das emissões de $\mathrm{CO}_{2}$ equivalente desses motores de acordo com quatro cenários distintos.

Para a evolução da frota de veículos com motores ciclo diesel, foram utilizados dados de emplacamento de veículos comerciais leves - LCT, caminhões médios - MDT e caminhões pesados - HDT disponibilizados nos anuários da Federação Nacional da Distribuição de Veículos Automotores - FENABRAVE. Além disso, foi considerado que todos os veículos vendidos têm que ser emplacados para transitar no país, logo, que o número de veículos emplacados passou a ser igual ao número de veículos vendidos. Posteriormente, fez-se a entrada de dados no modelo do número de veículos vendidos nos anos de 2000, 2005, 2010 e 2015, além de projeções futuras até 2050 de acordo com projeções do produto interno bruto - PIB desenvolvidas pela Empresa de Pesquisa Energética - EPE no Plano Nacional de Energia para 2050 - PNE 2050 e foi considerada uma taxa de obsolescência dessa frota proposta por Bartolo (2012).
Para as emissões de gases de efeito estufa, utilizou-se quatro cenários distintos: (i) expansão do biodiesel para o B20 até 2050 com uma formulação padrão de biodiesel, com $77 \%$ de óleo de soja, $19 \%$ de sebo bovino e $4 \%$ de óleo de algodão para produção do biodiesel; (ii) mesmo biodiesel, porém, com a expansão do biodiesel até B30 até 2050; (iii) biodiesel sem a adição de sebo bovino, logo, com $85 \%$ de óleo de soja e $15 \%$ de óleo de algodão para a sua fabricação e considerando uma expansão de biodiesel de B20; (iv) expansão para B30 e utilização do biodiesel sem a adição de sebo bovino. Sendo todos os biodieseis produzidos na fase inicial da produção descrito no item 4.1 e tendo seus dados de emissão de $\mathrm{CO}_{2 \mathrm{eq}}$ determinados conforme 4.3 deste trabalho.

Ademais, considerou-se que a expansão fosse feita de acordo com o Gráfico 2 e também, que a troca de diesel S50 para S10 ocorresse de acordo com o exposto por Vinhado (2017), evoluindo de 5,3\% em 2010, passando para $32,9 \%$ em 2015, chegando em 2020 no patamar de $39,7 \%, 50 \%$ em 2025 e finalizando em $90 \%$ em 2030 em diante, o que é condizente com o determinado por Bartolo (2012).

Por fim, foram analisados os dados de saída do modelo para os quatro cenários de consumo de combustível para motores de ciclo diesel, sendo diferenciada a utilização de diesel ou biodiesel, emissões de $\mathrm{CO}_{2 \mathrm{eq}}$ durante apenas o processo de queima do combustível e as emissões de $\mathrm{CO}_{2 \mathrm{eq}}$ de acordo com o well-totank do biodiesel. Logo, do processo de plantio até a chegada desse biocombustível no tanque do veículo, o que tornou possível a análise das reais vantagens e desvantagens da utilização de um ou de outro combustível. 


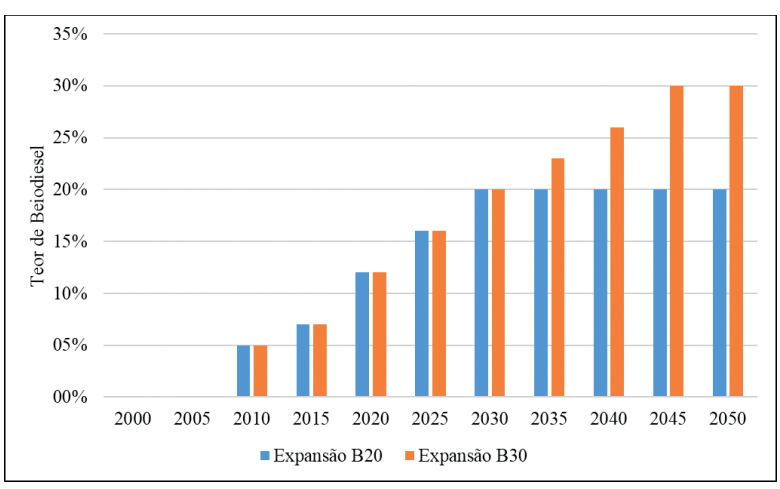

Gráfico 2. Propostas de expansão do teor de biodiesel (Fonte: Elaboração própria).

\section{Resultados e discussões}

\section{Características dos biodieseis}

$\mathrm{Na}$ caracterização físico-química, observou-se que todos os biodieseis obedeceram às normas da ANP, Resolução ANP n ${ }^{\circ}$ 45/2014 para os B100 e Resolução ANP $n^{\circ}$ 30/2016 para as concentrações BX até B30. Sendo possível afirmar que os combustíveis produzidos são biocombustíveis potencialmente comercializáveis no Brasil.

Com o auxílio da Tabela 2 foi possível analisar que não houve variações significativas entre os resultados dos biodieseis, identificando então que a produção do biodiesel com os sem sebo bovino não trouxe vantagens ou desvantagens significativas para essas duas características fundamentais do biodiesel, que são a viscosidade cinemática e a massa específica.

Entretanto, esses dois biodieseis produzidos (com e sem sebo bovino) apresentam diferenças quando comparamos as suas características de emissão de gases de efeito estufa. É possível observar na Tabela 3 que o biodiesel produzido sem sebo bovino possui emissões de $\mathrm{CO}_{2 \mathrm{eq}}$ inferior ao do biodiesel produzido com a presença de sebo bovino. Isso ocorrendo principalmente em concentrações superiores de biodiesel no diesel de petróleo, chegando a emitir cerca de $15 \%$ menos $\mathrm{CO}_{2 \mathrm{eq}}$ no $\mathrm{B} 30$ e $40 \%$ menos $\mathrm{CO}_{2 \mathrm{eq}}$ quando comparamos apenas os biodieseis puros (B100). Ainda, é possível analisar um comportamento diferente entre a evolução das emissões de cada concentração de biodiesel, sendo esse comportamento de elevação dos níveis de $\mathrm{CO}_{2 \mathrm{eq}}$ no biodiesel padrão (com sebo bovino) enquanto a concentração de biodiesel no diesel aumenta, o que já ocorre de maneira oposta no biodiesel sem sebo bovino, o qual apresenta uma diminuição dos níveis de $\mathrm{CO}_{2 \mathrm{eq}}$ conforme ocorre o aumento da quantidade de biocombustível no diesel.

É possível determinar que a retirada do sebo bovino do biodiesel não originou qualquer alteração significativa para as principais características físico-químicas do biodiesel, porém, auxiliou na redução dos níveis de $\mathrm{CO}_{2 \mathrm{eq}}$ da atmosfera, sendo extremamente para o combate as mudanças climáticas. Além disso, a retirada do sebo bovino do biodiesel apresentou uma característica positiva, se considerarmos que o gado durante o processo de desenvolvimento não fará qualquer retirada de carbono da atmosfera visto que o seu processo de digestão alimentar é responsável por uma grande quantidade de emissões de gases de efeito estufa na atmosfera. Já a soja e as outras oleaginosas de origem vegetal, durante o seu processo de desenvolvimento, fazem fotossíntese que retira carbono da atmosfera, fazendo com que haja uma diminuição dos níveis de emissão de $\mathrm{CO}_{2 \mathrm{eq}}$ durante o processo de well-to-tank, que será analisado posteriormente.

Tabela 2. Viscosidade cinemática e massa específica do biodiesel (Fonte: Elaboração própria)

\begin{tabular}{|c|c|c|c|}
\hline \multicolumn{2}{|c|}{ Biodiesel } & $\begin{array}{c}\text { Viscosidade } \\
\text { Cinemática }\left(\mathbf{m m}^{2} \cdot \mathbf{s}^{-2}\right)\end{array}$ & $\begin{array}{c}\text { Massa Específica } \\
\left(\mathbf{k g}^{-3}\right)\end{array}$ \\
\hline \multirow{4}{*}{ Padrão } & B8 & $2,96 \pm 0,15$ & $834,5 \pm 20,1$ \\
\hline & B10 & $2,99 \pm 0,30$ & $835,6 \pm 33,6$ \\
\cline { 2 - 4 } & B20 & $3,13 \pm 0,28$ & $840,9 \pm 27,3$ \\
\hline & B30 & $3,27 \pm 0,35$ & $846,1 \pm 34,9$ \\
\hline & B100 & $4,24 \pm 0,22$ & $883,1 \pm 28,3$ \\
\hline \multirow{4}{*}{$\begin{array}{c}\text { Sem Sebo } \\
\text { Bovino }\end{array}$} & B8 & $2,95 \pm 0,21$ & $834,5 \pm 20,3$ \\
\cline { 2 - 4 } & B10 & $2,98 \pm 0,36$ & $835,6 \pm 43,7$ \\
\hline & B20 & $3,11 \pm 0,34$ & $840,9 \pm 23,3$ \\
\hline & B30 & $3,24 \pm 0,29$ & $846,1 \pm 37,5$ \\
\hline & B100 & $4,14 \pm 0,30$ & $883,1 \pm 37,1$ \\
\hline
\end{tabular}

Tabela 3. Concentração de $\mathrm{CO}_{2 \mathrm{eq}}$ do biodiesel (Fonte: Elaboração própria).

\begin{tabular}{|c|c|c|}
\hline \multicolumn{2}{|c|}{ Biodiesel } & CO $_{2 \mathrm{eq}}{\mathbf{~ k g}, \mathbf{L g e}^{-1}}^{-1}$ \\
\hline \multirow{4}{*}{ Padrão } & $\mathrm{B} 8$ & $2,54 \pm 0,05$ \\
\hline & $\mathrm{B} 10$ & $2,55 \pm 0,16$ \\
\hline & B20 & $2,62 \pm 0,11$ \\
\hline & B30 & $2,70 \pm 0,13$ \\
\hline & B100 & $3,20 \pm 0,12$ \\
\hline \multirow{4}{*}{ Sem Sebo Bovino } & B8 & $2,44 \pm 0,05$ \\
\hline & B10 & $2,43 \pm 0,19$ \\
\hline & B20 & $2,38 \pm 0,09$ \\
\hline & B30 & $2,32 \pm 0,18$ \\
\hline & B100 & $1,96 \pm 0,08$ \\
\hline
\end{tabular}




\section{Modelagem MoMo Lite}

Com a inserção dos dados iniciais do modelo MoMo, foi possível verificar inicialmente que a frota de veículos apresentou uma tendência de redução de crescimento entre os anos de 2010 e 2015, chegando, no caso dos MDT e HDT, a decrescer o estoque total de veículos, Gráfico 3. Como pode ser visto no Gráfico 4, esse fato se deveu ao fato de no ano de 2015 ocorrer uma queda abrupta no número de vendas em todas as categorias de veículos movidos a diesel, sendo essa queda devido a grave crise financeira que o Brasil presenciou na época. Porém, nos anos seguintes passou a apresentar um ritmo de crescimento contínuo da frota, chegando no ano de 2040, no caso de MDT e HDT, a ultrapassar a quantidade de veículos da frota presente no ano de 2015. Já com os veículos LCT, como a redução das vendas em 2015 foi muito baixa, o estoque da frota não apresentou grandes reduções no número de veículos, apenas uma diminuição da taxa de crescimento dela entre os anos de 2025 e 2035.

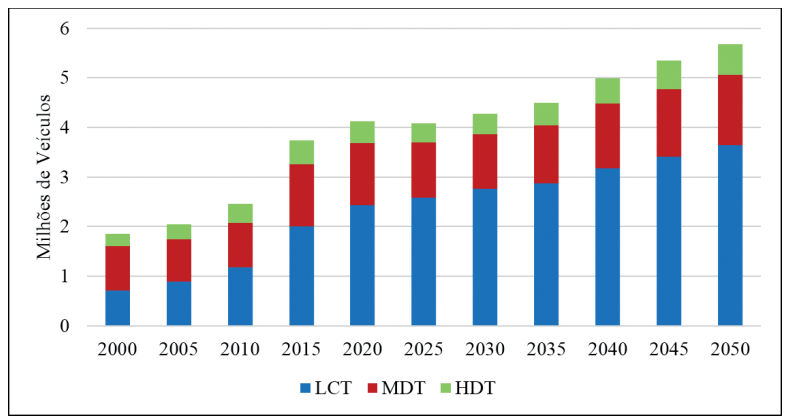

Gráfico 3. Evolução do estoque de veículos no Brasil (Fonte: Elaboração própria).

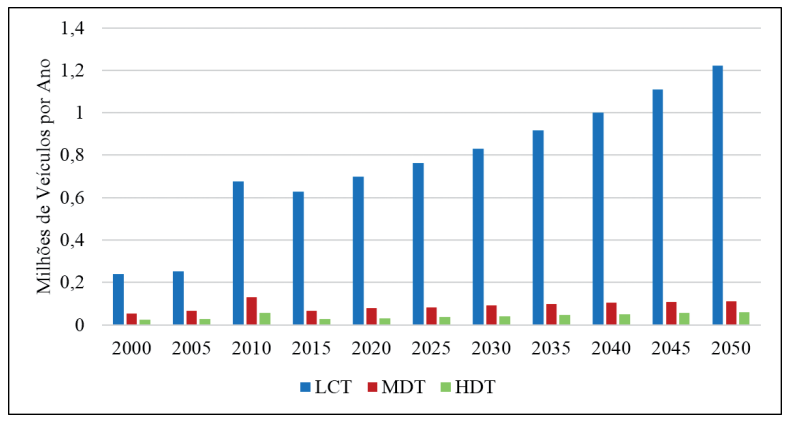

Gráfico 4. Evolução da venda de veículos no Brasil (Fonte: Elaboração própria).

Essa redução de frota também foi percebida no Gráfico 5 , onde se pode verificar o consumo esperado de combustível ao longo do tempo de estudo, o consumo de combustível apresentou aspectos muito similares aos apresentados pelo Gráfico 4. No caso dos veículos LCT, com leve redução no crescimento entre 2025 e
2035 e posterior retomada nas taxas de crescimento do consumo. Já com os veículos MDT e HDT, apresentando consumos menores do que os apresentados em 2015 entre os anos de 2020 e 2035 e posteriormente, elevação do consumo a partir do ano de 2040.

Outro ponto a se destacar no Gráfico 5, é a maior substituição do diesel de petróleo pelo biodiesel entre os anos de 2030 e 2050 em todos os cenários devido a política adotada de aumento da proporção de B20 para B30. Porém, o aumento diminuto do volume consumido de biodiesel entre os anos de 2040 e 2050 nos casos de adoção da política de aumento das proporções de biodiesel até no máximo B20, apesar do aumento significativo do consumo de combustível diesel durante esse período. Este fato já não acontece quando observamos os casos de aumento da proporção de biodiesel até B30.

Sendo esse ponto interessante divido a necessidade de aumento de produção de oleaginosas como soja e semente de algodão, trabalhadas nesse estudo. Além da necessidade da adoção de outras políticas como a do aumento da diversidade de fontes vegetais para comportar esse aumento expressivo do.

volume de biodiesel. Esse debate é importante principalmente quando há a exclusão do sebo bovino, que faz com que o pensamento inicial do PNPB de aproveitamento do potencial produtivo de cada região volte à tona, visto que essa grande demanda de biocombustível necessita de uma fonte constante, barata e não degradante, que só é possível de ser alcançada com o estudo do tipo ideal de cultura de oleaginosa adequada para o solo e clima de cada região.

A adoção de novas fontes triglicerídeas de origem vegetal mais bem adaptada para cada parte do território brasileiro no cenário de exclusão do sebo bovino do biodiesel é importante devido a impossibilidade da contínua a expansão das fronteiras agrícolas nos territórios adequados para a produção da soja, que é a principal fonte de matéria-prima do biodiesel brasileiro. Assim como acontece com a soja, o algodão necessitará de condições edafoclimáticas específicas para o seu desenvolvimento. Além disso, o óleo de algodão possui valor comercial muito superior a outros óleos, como o de palma, que já é utilizado em grande escala para a produção de biocombustível na Oceania, visto suas propriedades farmacêuticas, assim como o óleo de coco. Com o auxílio do Gráfico 6 fica evidente que a remoção do sebo bovino do biodiesel é mais importante, do ponto de visto ambiental, do que o aumento da proporção de 
biodiesel no diesel durante o processo de queima desse combustível, visto que a simples eliminação dessa matéria-prima propiciou a redução de $10 \%$ nas taxas de emissão de $\mathrm{CO}_{2 \mathrm{eq}}$. Além disso, é possível observar que ao contrário do que é esperado, o aumento da proporção de biodiesel, com sebo bovino em sua composição, no diesel de petróleo proporcionou o aumento das taxas de emissão de $\mathrm{CO}_{2 \mathrm{eq}}$ durante a fase de queima. Isso mostra que dependendo das concentrações, o biodiesel de sebo bovino pode ser pior para o meio ambiente do que o próprio diesel.

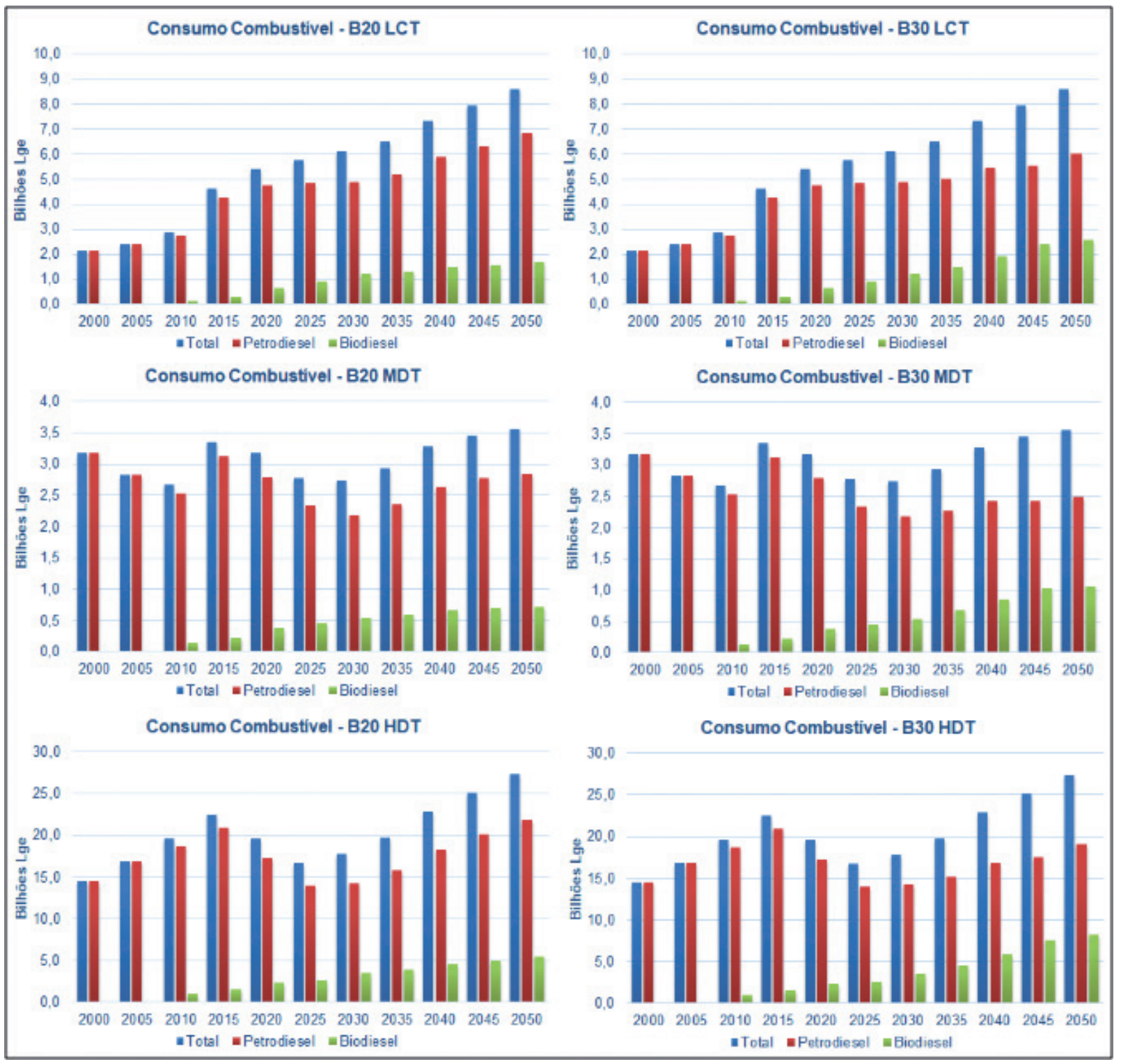

Gráfico 5. Evolução do consumo de diesel e biodiesel no Brasil (Fonte: Elaboração própria).

Entretanto, do ponto de vista ambiental, a utilização do sebo para a produção de biodiesel é importante visto a falta de alternativas viáveis para o aproveitamento deste material na indústria. Com isso, se tornam necessários estudos de possíveis destinos para ele de forma a evitar que ele seja descartado em aterros sanitários. Porém, no longo prazo, essa quantidade que matéria-prima animal deve apresentar diminuição devido à conscientização da importância da troca da dieta alimentar com o intuito de redução dos impactos ambientais causados pela emissão de metano $\left(\mathrm{CH}_{4}\right)$ (IPCC, 2019). Esta modificação muitas vezes acaba sendo uma troca entre a quantidade pela qualidade, tanto no nível gustativo, quanto no nutricional (EISLER et al., 2014). Portanto, sendo possível que em cenários futuros sejam observadas menores quantidade de carcaças bovinas e consequentemente, menos sebo bovino. 


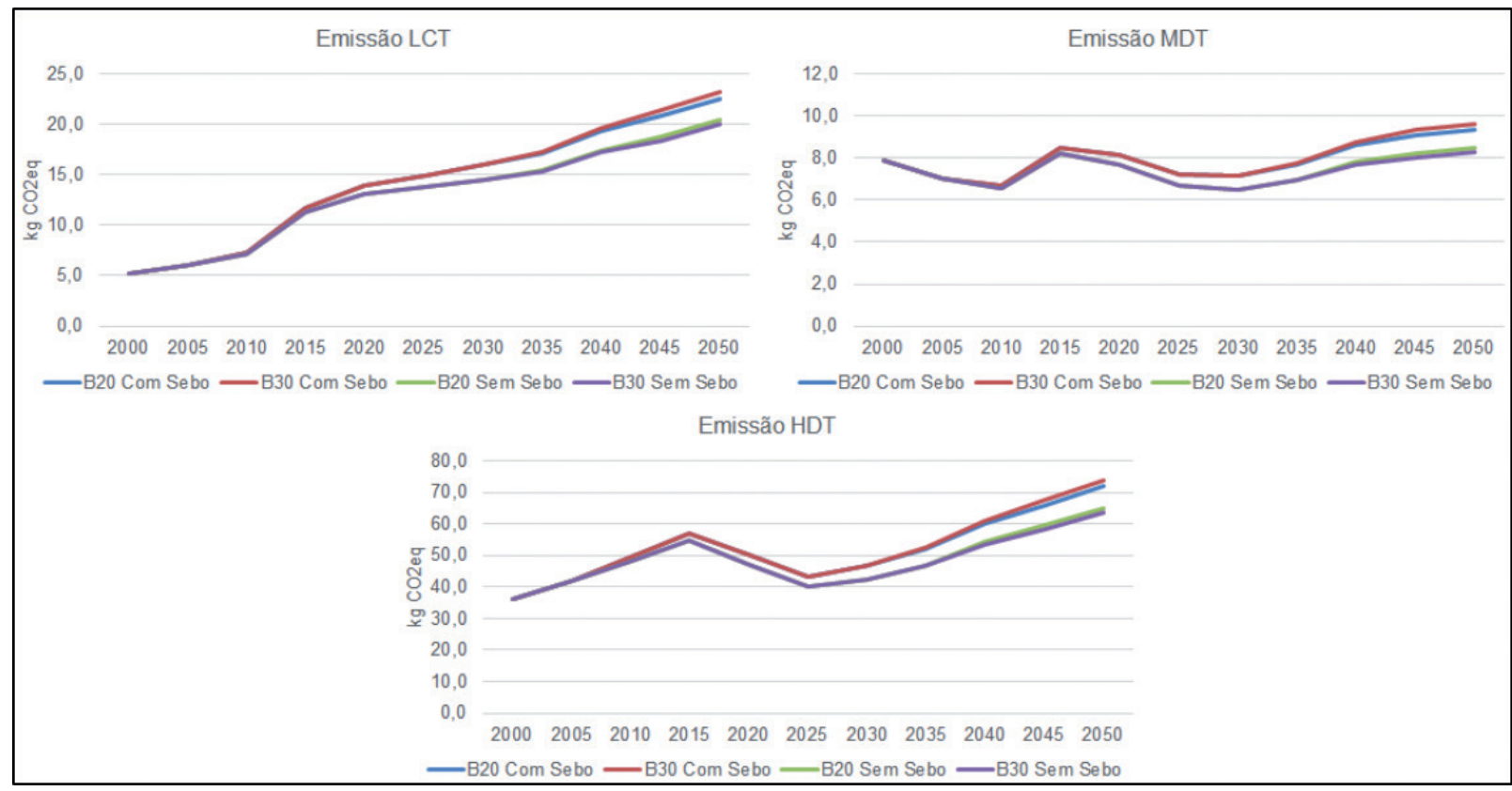

Gráfico 6. Emissões de $\mathrm{CO}_{2 \mathrm{eq}}$

(Fonte: Elaboração própria).

A importância da substituição do sebo bovino também se torna clara no Gráfico 7 que apresenta as emissões dos GEE do well-to-tank, logo, desde o processo inicial da criação da matéria-prima até chegar como biodiesel no tanque de combustível do veículo. Nesse caso, pode-se perceber que a simples retirada do sebo bovino propiciou o aumento da retirada de $\mathrm{CO}_{2 \mathrm{eq}}$ produzido durante o processo de cultivo da matéria-prima e produção do biodiesel de $28 \%$, fazendo com que o consumo de $\mathrm{CO}_{2 \mathrm{eq}}$ pela planta durante o seu processo de desenvolvimento fosse superior a emissão de $\mathrm{CO}_{2 \mathrm{eq}}$ durante as fases posteriores do ciclo do biodiesel.

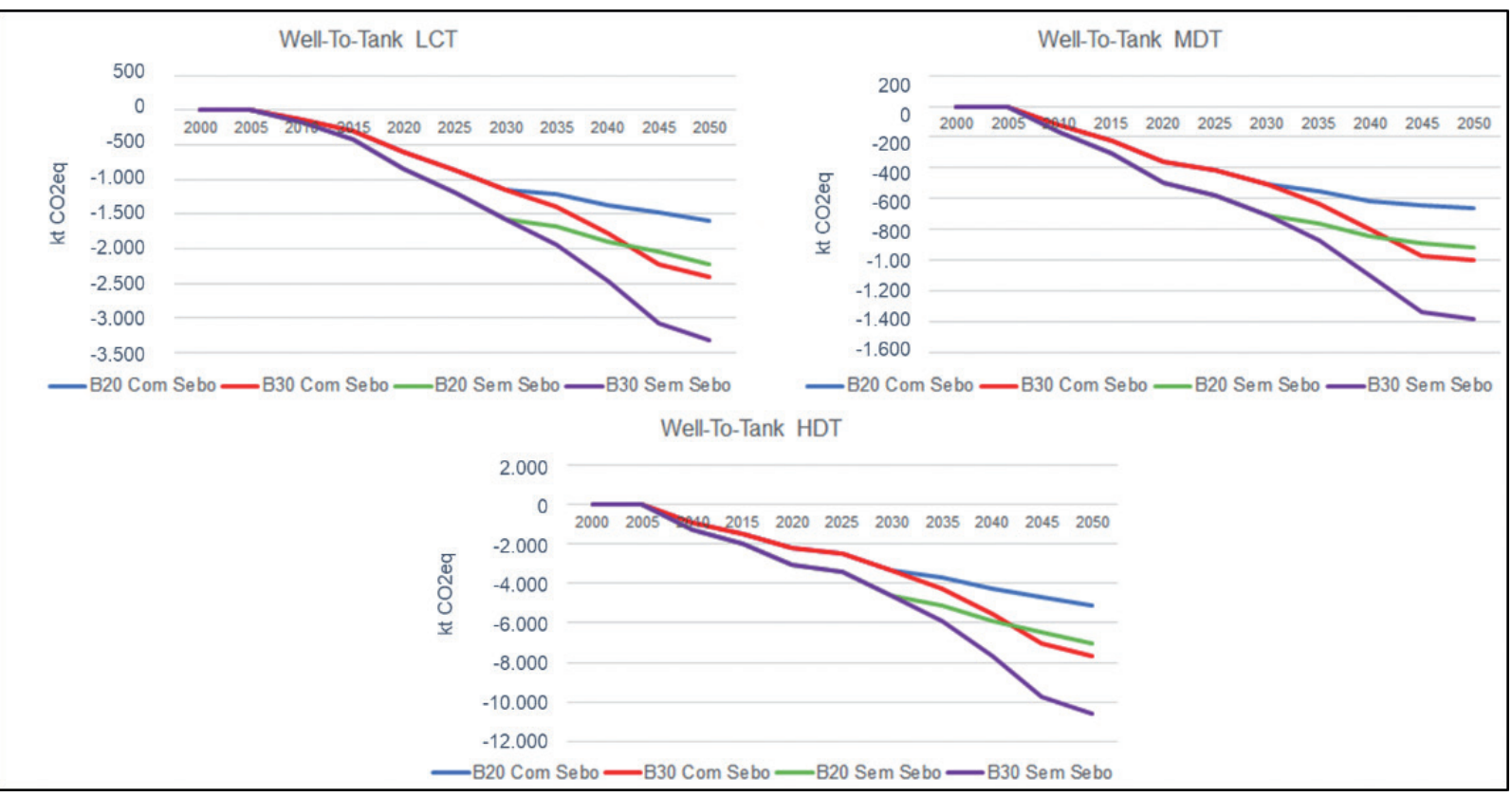

Gráfico 7. Emissões de $\mathrm{CO}_{2 \mathrm{eq}}$ sob a ótica well-to-tank

(Fonte: Elaboração própria). 
Além disso, outro fator muito importante para a análise well-to-tank exposto pela modelagem foi a importância do aumento da proporção de biodiesel no diesel, que anteriormente, na fase de queima, não apresentava reduções muito significativas das taxas de $\mathrm{CO}_{2 \mathrm{eq}}$ e nessa análise já se mostrou mais vantajosa do que a eliminação do sebo bovino como fonte de matériaprima para o biodiesel. A mudança de B20 para B30 foi responsável pelo aumento de $34 \%$ da retirada de $\mathrm{CO}_{2 \mathrm{eq}}$ durante o processo de produção, o que não pode ser observado anteriormente ao analisarmos a queima desses combustíveis. Logo, no caso da necessidade de adoção de apenas uma das duas alternativas no longo prazo, sendo mais interessante o desenvolvimento de uma política de aumento da proporção de biodiesel no diesel do que a substituição dessa fonte triglicerídea por outra de origem vegetal. Porém, tendo a possibilidade, o gráfico anterior mostra que a adoção das duas medidas é extremamente positiva, visto que a diferença de remoção de $\mathrm{CO}_{2 \mathrm{eq}}$ entre o $\mathrm{B} 20$ com sebo bovino e o B30 sem sebo bovino é de $52 \%$ e seria responsável, no caso da junção de todos os veículos estudados, pela remoção de cerca de 8 bilhões de $\mathrm{kg}$ de $\mathrm{CO}_{2 \mathrm{eq}}$ a mais do meio ambiente.

\section{Conclusão}

A adoção de uma política de aumento do percentual de biodiesel no diesel é uma alternativa ambientalmente viável, visto a significativa diminuição dos níveis de emissão de gases de efeito estufa e, devido à grande captura de carbono que é feita durante o processo de desenvolvimento das oleaginosas.

No trabalho, concluiu-se que é possível a adoção de proporções superiores de biodiesel no diesel de petróleo as descritas na literatura como máximas (B20), porém, tomando-se o cuidado de utilizar uma maior quantidade de fontes oleaginosas que produzam biodieseis com menores viscosidades cinemáticas e com ponto de entupimento de filtro a frio mais baixos, como foi o caso do B30 de óleo de soja e de semente de algodão. Também, que é necessário haver a diversificação da matéria-prima de origem vegetal, assim como o melhor aproveitamento do potencial agrícola dessas fontes, conforme o que no pensado inicialmente durante o período de implementação do PNPB.

Além disso, também pode-se concluir que a substituição de matérias-primas não renováveis, como é o caso do sebo bovino, por fontes renováveis de origem vegetal é de suma importância, tendo em vista que o aumento da porcentagem dessas fontes vegetais propiciará o gradativo aumento da captura de gases de efeito estufa. No caso da soja que além de utilizar o $\mathrm{CO}_{2}$ para o seu crescimento, ainda é responsável pela fixação de nitrogênio no solo, já que ela é uma leguminosa, o que auxilia na nutrição natural do solo. Porém, sendo necessário adotar a rotação de cultura para que não ocorram processos de degradação do solo já conhecidos da monocultura, como a retirada excessiva de certos nutrientes do solo, proliferação de pragas mais resistentes e a necessidade de aumentar os gastos devido a adoção de grandes quantidades de fertilizantes e defensivos agrícolas.

Também, mostrou-se a necessidade de haver uma análise mais ampla da cadeia produtiva do biodiesel, do tipo well-to-tank ou well-to-wheel, para afirmar que uma matéria-prima é superior ou inferior a outra. Isso se devendo ao fato de que cada fonte triglicerídea necessitará de diferentes compostos e processos durante a sua produção, podendo fazer com que um biodiesel que emita menos $\mathrm{CO}_{2 \mathrm{eq}}$ durante o processo de queima, seja responsável por um maior aporte de gases de efeito estufa durante o seu ciclo de produção, tornando-o menos interessante do ponto de vista ambiental.

\section{Referências bibliográficas}

1. ABIOVE, APROBIO, UBRABIO. (2016). Biodiesel: Oportunidade e Desafios no Longo Prazo. Brasília.

2. Amaris, J. M., Manrique, D. A., \& Jaramillo, J. E. (2015). Biocombustibles líquidos en Colombia y su impacto en motores de combustión interna. Una revisión. Revista Fuentes, 13(2), 23-34.

3. Angelkorte, G. B. (2016). Eficiência na Produção de Biodiesel a Partir de Misturas Prévias de Óleos Vegetais. Universidade Federal Fluminense, Trabalho de Conclusão de Curso.

4. ANP - Agência Nacional de Petróleo, Gás Natural e Biocombustíveis. (2014). Resolução ANP $n^{o}$ 45/2014 - Informações aos Agentes Econômicos que Comercializam Biodiesel. Brasil, Brasília.

5. ANP - Agência Nacional de Petróleo, Gás Natural e Biocombustíveis. (2016). Resolução ANP $n^{o}$ 30/2016. Brasil, Brasília.

6. ANP - Agência Nacional de Petróleo, Gás Natural e Biocombustíveis. (2019). Biodiesel. Brasil, Brasília. Disponível em: http://www.anp.gov. br/biocombustiveis/biodiesel.

7. Ayala, F. E. B., Gómez, J. Q., \& León, E. A. (2011). Estudio de factibilidad del uso del biodiesel como fase continua en lodos de perforación de emulsión inversa. Revista Fuentes, 9(1).

8. Bartolo, T. D. (2012). Avaliação da Factibilidade da Política de Padronização Veicular da união 
Europeia, a Partir da Maior Inserção do Etanol Brasileiro. Dissertação de Mestrado, Programa de Planejamento Energético, COPPE/UFRJ.

8. Brasil. (2015). PL. 3.834/2015. Brasília, Brasil.

9. Brasil. (2016). Boletim Mensal dos Combustiveis Renováveis. Ministério de Minas e Energia MME, Secretaria de petróleo, Gás Natural e Combustíveis Renováveis, Departamento de Combustíveis Renováveis - DCR, Brasília, Ed. 96.

10. Cherubini, F. (2010). GHG Balances of Bioenergy Systems - Overview of Key Steps in the Production Chain and Methodological Concerns. Renewable Energy, Vol. 35, Issue 7, p. 1565-1573.

11. CNPE - Conselho Nacional de Política Energética. (2015). Resolução CNPE $n^{o}$ 3/2015. Brasília, Brasil.

12. Coronado, C. R., Carvalho Jr, J. A., Silveira, J. L. (2009). Biodiesel CO2 Emissions: A Comparison with the Main Fuels in the Brazilian Market. FUEL PROCESSING TECHNOLOGY 90, p. 204-211.

13. Costa, A. O. (2017). A Inserção do Biodiesel na Matriz Energética Nacional: Aspectos Socioeconômicos, Ambientais e Institucionais. Tese de Doutorado, Programa de Planejamento Energético, COPPE/UFRJ, Rio de Janeiro.

14. Eisler, M. C., Lee, M. RF., Tarlton, J. F., Martin, G., Beddington, J., Dungait, J. A. J., ... Winter, M. (2014). Steps to sustainable livestock: With improved breeding and cultivation, ruminant animals can yield food that is better for people and the planet. Nature, 507(7490), 32-34. https://doi.org/10.1038/507032a

15. EPE - Empresa de Pesquisas Energéticas. (2014) Plano Nacional de Energia 2050. Empresa de Pesquisas Energéticas - EPE, Rio de Janeiro.

16. FENABRAVE - Federação Nacional da Distribuição de Veículos Automotores. (2000). Anuário 2000 - O Desempenho da Distribuição Automotiva no Brasil., São Paulo.

17. FENABRAVE - Federação Nacional da Distribuição de Veículos Automotores. (2005). Anuário 2005 - $O$ Desempenho da Distribuição Automotiva no Brasil. São Paulo.

18. FENABRAVE - Federação Nacional da Distribuição de Veículos Automotores. (2010). Anuário 2010 - O Desempenho da Distribuição Automotiva no Brasil. São Paulo.

19. FENABRAVE - Federação Nacional da Distribuição de Veículos Automotores. (2015). Anuário 2015 - O Desempenho da Distribuição
Automotiva no Brasil. São Paulo.

20. Gnansounou, E. (2009). Fuel Ethanol - Current Status and Outlook. Em: PANDEY, A. Handbook of Plant-Based Biofuels. Estados Unidos da América: Taylor \& Francis Group, Cap. 5.

21. Harold, S. (1997). Industrial Vegetable Oils: Opportunities Within the European Biodiesel and Lubrificant Markets. Part 2. Market Characteristics. Lipd Technol, Ed.10, p. 67-70.

22. IPCC - Intergovernmental Panel on Climate Change. (2019). IPCC Special Report on Climate Change, Desertification, Land Degradation, Sustainable Land Management, Food Security, and Greenhouse gas fluxes in Terrestrial Ecosystems - Summary for Policymakers. Working Group III (WGIII) - Mitigation of Climate Change. London, UK.

23. Jewett, B. (2003). Biodiesel Powers Up. Inform ed.14, p. 528-530.

24. Knothe, G.; Gerpen, J. V.; Krahl, J.; Ramos, L. P. (2006). Manual de Biodiesel. São Paulo, Edgard Blucher.

25. Masjuki, H. H., Sapuan, S. M. (1995). Palm Oil Methyl Esters as Lubricant Additives in Small Diesel Engines. Ibid., Ed. 72, p. 609-612.

26. Nitske, W. R., Wilson, C. M. (1965). Rudolf Diesel, Pioneer of the Age of Power. University of Oklahoma Press, Norman, Oklahoma.

27. Rosa, L. P., Oliveira, L. B., Costa, A. O., Pimenteira, C. A. P., Mattos, L. B. R., Henriques, R. M., Aranda, D. A. (2003). Geração de Energia a Partir de Resíduos Sólidos Urbanos e Óleos Vegetais. In: Proceedings of Tolmasquim, M. T. (Coord) Fontes Alternativas de Energia no Brasil, CENERGIA, Ed 1, Editora Interciência, p.515.

28. Salomão, I. L. (2013). Análise do Programa Nacional de Produção e Uso do Biodiesel no Brasil entre os Anos 2005 e 2010: O Papel Dominante do Biodiesel de Soja. Dissertação de Mestrado, Programa de Planejamento Energético, COPPE/UFRJ.

29. Singh, S. P., Singh, D. (2010). Biodiesel Production Through the Use of Different Sources and Characterization of Oils and Their Esters as the Substitute of Diesel: A review. Renewable and Sustainable Energy Reviews, Ed. 14, p. 200-216.

30. Tomasevic, A. V., Marinkovic, S. S. (2003). Methanolysis of Used Frying Oils. Fuel Process Technol, Ed. 81, p. 1-6.

31. Uribe, R. A. M., Alberconi, C. H., Taveres, B. A. (2014). Produção de Biodiesel a Partir do Sebo Bovino: Viabilidade Econômica e Métodos de 
produção. Congresso Nacional de Excelência em Gestão - CNEG, Rio de Janeiro.

32. Villela, A. (2014). Expansão da Palma na Amazônia Oriental para Fins Energéticos. Programa de Planejamento Energético, COPPE, da Universidade Federal do Rio de Janeiro, p. 388.
33. Vinhado, F. S. (2017). Combustiveis: Especificação/ Fornecimento. Agência Nacional de Petróleo, Gás Natural e Biocombustíveis - ANP, Brasília.

34. WHO - World Health Organization. (2014). 7 Million Premature Deaths Annually Linked to Air Pollution.

Recepción: 10 de julio de 2019

Aceptación: 17 de diciembre de 2019 